\title{
Editorial
}

\section{Clinical relevance of right ventricular dysplasia/cardiomyopathy}

(Arrhythmogenic) right ventricular dysplasia, also called (arrhythmogenic) right ventricular cardiomyopathy $(\mathrm{RVD} / \mathrm{C})$ is a recently defined heart muscle disease of unknown origin that predominantly, although not exclusively, affects the right ventricular myocardium.

Its pathological hallmark is the atrophy of myocytes with fatty or fibro-fatty infiltration of the right ventricle. ${ }^{1}$ The typical clinical picture is related to ventricular tachyarrhythmias with left bundle branch block morphology, which may cause sudden death, ${ }^{12}$ while a less frequent presentation is "right" heart failure. The disease may be localised or widespread, with biventricular involvement in some cases. ${ }^{3}$

In the past decade several investigators have contributed to the knowledge of this "new" cardiomyopathy ${ }^{4}$; nevertheless, there are still many uncertainties that deserve further studies. This editorial is mainly oriented to some still unsolved problems of RVD/C.

\section{Aetiologic theories}

Several hypotheses have been advocated to explain the cause and pathogenesis of RVD/C. The first, that explains the term "dysplasia" (from the ancient Greek, abnormality of development) considered the disease a congenital or disontogenetic disorder caused by an abnormal development of right ventricular myocardium. ${ }^{2}$ However, clinical and pathological observations favour the concept of a degenerative process ("atrophy" or "dystrophy") caused by progressive death of myocardial cells. ${ }^{5}$ Recent reports suggest that programmed myocyte death (apoptosis) may play an important role, and Mallat et al observed this process in the right ventricular myocardium of patients with RVD/C. ${ }^{6}$ Moreover, James et al demonstrated massive apoptosis at the level of the right ventricle and conductive system in an infant with Uhl's disease. ${ }^{7}$

Several factors, known or suspected to be present in the failing myocardium (cytokines, oxidants, nitric oxide, growth factors), can stimulate apoptosis in a variety of cells including cardiac myocytes. However, it is speculative whether apoptosis reflects a primary hereditary abnormality that determines an imbalance between the stimulatory and inhibitory mechanisms that physiologically regulate this process.

The frequent familial incidence (present in at least one third of cases of RVD/C), with an autosomal dominant hereditary transmission, ${ }^{8}$ is consistent with a genetic disease. Using molecular genetic techniques and linkage analysis Rampazzo and colleagues ${ }^{9}$ mapped the first genetic linkage marker for RVD/C to the long arm of chromosome 14. More recently other chromosomal localisations have been found in other families. ${ }^{10}$ These data support the existence of a genetic heterogeneity. The putative disease genes are still unknown and are currently under investigation. ${ }^{10}$

The last hypothesis, particularly attractive for sporadic cases, is that an inflammatory process may have a dominant role in the pathogenesis of the disease. The inflammatory theory is supported by the demonstration of inflammatory infiltrates at endomyocardial biopsy or necropsy, ${ }^{5}$ sometimes compatible with an active myocarditis. ${ }^{11}$ However, whether myocarditis is a primary
Table 1 Clinical and ECG findings useful for diagnosing right ventricular dysplasia/cardiomyopathy $(R V D / C)$

Familial history of RVD/C or premature sudden death

Clinical presentation

Ventricular arrhythmias (sometimes sudden death)

Right congestive heart failure

Supraventricular arrhythmias

Asymptomatic with abnormal ECG, chest $x$ ray, or echocardiography

No specific physical findings (widely split II heart sound)

ECG abnormalities

$\mathrm{T}$ wave inversion on right precordial leads

$\varepsilon$ waves (late potentials)

Prolonged QRS duration on V1-V2

Characteristics of ventricular arrhythmias

Frequently with LBBB pattern (right ventricular origin)

Wide range of severity (from patient to patient and during the course of disease) from sustained or non-sustained VT (rarely VF), to ventricular ectopic beats

LBBB, left bundle branch block; VT, ventricular tachycardia; VF, ventricular fibrillation.

process in $\mathrm{RVD} / \mathrm{C}$ or a phenomenon superimposed on a myopathic heart is still a source of speculation. ${ }^{5}$ The definition of the aetiology and pathogenesis of the disease is even more problematic in some cases in which familiarity, myocarditis, myocyte loss, and fat substitution are associated. ${ }^{11}$

Spectrum of clinical and structural abnormalities

$\mathrm{RVD} / \mathrm{C}$ may have different clinical presentations (table 1). The classic clinical picture is characterised by sustained or non-sustained ventricular tachycardia with left bundle branch morphology, suggesting an origin from the right ventricle. ${ }^{2}$ In some cases severe ventricular arrhythmias may cause cardiac arrest and sudden death. In fact, in a large postmortem study ${ }^{1} \mathrm{RVD} / \mathrm{C}$ was a leading cause of sudden death in the young. In other patients supraventricular arrhythmias or congestive heart failure are the dominant clinical findings. ${ }^{3}{ }^{12}$ Severe right heart failure may be observed in infancy in Uhl's anomaly, a congenital disease characterised by a widespread right ventricular atrophy with a parchment-like right ventricle; whether this disease is part of the spectrum of RVD/C is as unsolved question. ${ }^{13}$

There are no specific physical signs with clinical examination, and often the severity of structural abnormalities contrasts with the relatively mild and subtle physical findings. A recognised physical sign is a widely split second heart sound, but this is seldom reported. It occurs presumably as a consequence of right ventricular dysfunction. ${ }^{2}$ The ECG shows abnormalities in $75 \%$ of cases, such as $\mathrm{T}$ wave inversion in the right precordial leads or right ventricular activation delay, which are per se not diagnostic, but in young subjects with ventricular arrhythmias they may be an important contribution to diagnosis. Late potentials are frequently present with signal averaged ECG, and sometimes also evident on the surface ECG (epsilon waves). ${ }^{2}$

A spectrum of right ventricular structural abnormalities are described in RVD/C (table 2), ranging from wall aneurysms, typically located within the triangle of dysplasia (apex, outflow, and subtricuspid area), ${ }^{2}$ to diffuse dilatation of the chamber. Several imaging techniques, such as cross sectional echocardiography, contrast and radionuclide 
Table 2 Diagnostic methods for evaluation of structural and functional ventricular abnormalities in right ventricular dysplasia/cardiomyopathy $(R V D / C)$

Echocardiography (diagnostic method of choice in most cases)

RV asynergic bulges or aneurysms ("triangle of dysplasia": outflow, apex, and subtricuspid area)

RV regional or global enlargement with depressed systolic function

LV normal or with regional asynergic areas (rarely aneurysms)

LV systolic dysfunction (mild to moderate, rarely severe), usually without significant enlargement

TR with normal jet velocity at Doppler study

Cardiac MRI (selected cases, particularly when non-diagnostic

echocardiography findings)

Spin-echo technique

non-invasive myocardial tissue characterisation

hyperintense areas $>$ fatty infiltration

Cine-MRI technique

RV regional or global enlargement, systolic dysfunction, wall aneurysms RV endomyocardial biopsy (very selected cases)

May be diagnostic, allowing in vivo demonstration of RV myocardial atrophy and fatty or fibro/fatty substitution

Invasive, potentially dangerous (risk of perforation of thin atrophic wall) False negative cases (localised disease)

$\mathrm{LV}$, left ventricle; RV, right ventricle; TR, tricuspid regurgitation; MRI, magnetic resonance imaging.

angiography, computed tomography, and magnetic resonance imaging (MRI) can provide information regarding these structural abnormalities and have been employed in several studies. In clinical practice, echocardiography is the initial screening test used in diagnosing RVD/C; however, its sensitivity and specificity are not very high, particularly in patients with suboptimal image quality and subtle abnormalities. In these cases, MRI appears to be the best imaging technique, as it provides accurate information on volumes, wall motion right ventricular abnormalities, and abnormal tissue composition of walls. ${ }^{14}$ Using spin-echo MRI, fatty infiltration of the right ventricle can be identified as an intense signal compared with the surrounding myocardium. ${ }^{14} 15$

Some years ago our group demonstrated that RVD/C, initially considered a disease strictly confined within the right ventricle, frequently involves the left ventricle as well. ${ }^{3}$ The clinicopathological data of a recent multicentre study ${ }^{16}$ support the concept that the disease frequently involves both ventricles, particularly in more advanced and progressive cases.

Contrary to dilated cardiomyopathy, RVD/C with biventricular involvement is usually characterised by disproportionate right ventricular enlargement and dysfunction, and by the presence of wall bulges, whereas the left ventricle is only mildly dilated, although it can be severely hypokinetic in some cases. ${ }^{14}{ }^{16-18}$

Because of the diagnostic difficulties, a set of diagnostic criteria for RVD/C were recently proposed by the task force of the working group on "myocardial and pericardial disease" of the European Society of Cardiology and the Scientific Council of Cardiomyopathies of the International Society and Federation of Cardiology, which distinguished "major" and "minor" criteria. ${ }^{8}$ Although a clinical validation of the proposed criteria on large patient series is not presently available, preliminary data confirm their diagnostic impact. ${ }^{19}$ The association of the suggested diagnostic criteria is a major step in the clinical diagnosis of $\mathrm{RVD} / \mathrm{C}$ and must be used in clinical practice.

In most cases the confirmation of diagnosis by endomyocardial biopsy of the right ventricle is not required. It is possible using endomyocardial biopsy to demonstrate the typical, highly diagnostic, histological abnormalities of $\mathrm{RVD} / \mathrm{C},{ }^{20}$ but it is an invasive procedure, with potential risk of perforation and cardiac tamponade, considering the thin and atrophic right ventricular walls of these patients; thus this procedure has to be used in very selected controversial cases.
Natural history, prognosis, and treatment

RVD/C was essentially considered an arrhythmogenic disease of young people, but recent data suggest a more extensive spectrum of possibilities. First, the true onset of the disease is unknown, as a concealed phase probably precedes the symptomatic phase. In contrast, some patients with advanced RVD/C can develop severe heart failure that mimics dilated cardiomyopathy. ${ }^{16-18}$ The available long term follow up studies of these patients are relatively few, and they may have selection bias. In fact, most of them considered only patients with severe arrhythmias, ${ }^{21}{ }^{22}$ and postmortem studies on RVD/C focused on sudden death of young subjects.

Among different series ${ }^{122122}$ a progression of the disease was frequently reported, although some patients may have stable symptoms for many years. Recent data from the multicentre clinicopathology study ${ }^{16}$ confirm the hypothesis that RVD/C has to be considered a progressive disease with frequent biventricular involvement leading to death from heart failure.

The two most frequent causes of death in patients with RVD/C are severe ventricular arrhythmias, and progressive heart failure, with a similar expected rate of approximately $1 \%$ per year. ${ }^{13}$ In the major reported studies ${ }^{12} 2122$ mortality rates were variable, ranging from $4 \%$ to $20 \%$ for similar follow up times. The risk of sudden death is difficult to predict, ${ }^{21}$ and possible prognostic factors are history of syncope and complex ventricular arrhythmias. ${ }^{22}$ Electrophysiologically guided serial drug testing can identify effective antiarrhythmic drug treatment in most patients, and sotalol has been shown to be the drug of choice for the control of these arrhythmias. ${ }^{23}$ Non-pharmacological treatment, mainly implantable defibrillators, is indicated for patients with RVD/C and severe ventricular arrhythmias in whom drug treatment is ineffective or associated with significant side effects. ${ }^{24}$ Furthermore, implantable defibrillators are advisable when drug effects are uncertain owing to the fact that baseline electrophysiological studies after an episode of reversible cardiac arrest do not induce significant ventricular arrhythmias. ${ }^{24}$

The problem of heart failure is not negligible in RVD/C. In fact, in our series, heart failure, usually observed in patients with severe biventricular dysfunction, was the most frequent cause of death and reason for heart transplantation. ${ }^{22}$ As in patients with dilated cardiomyopathy, heart transplantation is indicated for refractory heart failure or life threatening ventricular arrhythmias.

\section{Conclusions and future directions for research}

$\mathrm{RVD} / \mathrm{C}$ is a recently recognised cardiomyopathy, which has several important aspects of clinical relevance. They are mainly related to its peculiar pathological substrate, to the uncertainties about its aetiology and pathogenesis, to the difficulties in making a clinical diagnosis, and to the still incomplete knowledge about evolution, prognosis, and treatment. Large prospective multicentre studies are advisable to improve our knowledge of this fascinating disease.

B PINAMONTI G SINAGRA

F CAMERINI

Divisione di Cardiologia, Ospedale Maggiore, piazza Ospedale 1, 34129 Trieste, Italy

1 Thiene G, Nava A, Corrado D, et al. Right ventricular cardiomyopathy and sudden death in young people. N Engl F Med 1988;318:129-33.

2 Marcus FI, Fontaine GH, Guiraudon G, et al. Right ventricular dysplasia: a report of 24 adult cases. Circulation 1982;65:384-98.

3 Pinamonti B, Sinagra G, Salvi A, et al. Left ventricular involvement in right ventricular dysplasia. Am Heart f 1992;123:711-24.

4 Richardson P, McKenna W, Bristow M, et al. Report of the 1995 World Health Organization/International Society and Federation of Cardiology task force on the definition and classification of cardiomyopathies. Circulatask force on the def
tion $1996 ; 93: 841-2$. 
5 Basso C, Thiene G, Corrado D, et al. Arrhythmogenic right ventricular cardiomyopathy. Dysplasia, dystrophy, or myocarditis? Circulation 1996;94: diomyop

6 Mallat Z, Tedgui A, Fontaliran F, et al. Evidence of apoptosis in arrhythmogenic right ventricular dysplasia. $N$ Engl f Med 1996;335:11906.

7 James TN, Nichols MM, Sapire DW, et al. Complete heart block and fatal right ventricular failure in an infant. Circulation 1996;93:1588-1600.

8 McKenna WJ, Thiene G, Nava A, et al. Diagnosis of arrhythmogenic right ventricular dysplasia/cardiomyopathy. Br Heart $\mathcal{F}$ 1994;71:215-18.

9 Rampazzo A, Nava A, Danieli GA, et al. The gene for arrhythmogenic right ventricular cardiomyopathy maps to chromosome 14q23-q24. Hum Mol Genet 1994;3:959-62.

10 Mestroni L, Giacca M. Molecular genetics of dilated cardiomyopathy. Curr Opin Cardiol 1997;12:303-9.

11 Pinamonti B, Miani D, Sinagra GF, et al. Familial right ventricular dysplasia with biventricular involvement and inflammatory infiltration. Heart 1996;76:66-9.

12 Kullo IJ, Edwards WD, Seward JB. Right ventricular dysplasia: the Mayo Clinic experience. Mayo Clin Proc 1995;70:541-8.

13 Fontaine G. Arrhythmogenic right ventricular dysplasia. Curr Opin Cardiol 1995;10:16-20

14 Ricci C, Longo R, Pagnan L, et al. Magnetic resonance imaging in right ventricular dysplasia. Am f Cardiol 1992;70:1589-95.

15 Menghetti L, Basso C, Nava A, et al. Spin-echo nuclear magnetic resonance for tissue characterisation in arrhythmogenic right ventricular cardiomyopathy. Heart 1996;76:467-70.
16 Corrado D, Basso C, Thiene G, et al. The spectrum of clinico-pathologic manifestations of right ventricular cardiomyopathy/dysplasia. A multimanifestations of right ventricular cardiomyop
center study. 7 Am Coll Cardiol 1997;30:1512-20.

17 Miani D, Pinamonti B, Bussani R, et al. Right ventricular dysplasia: a clinical and pathological study of two families with left ventricular involvement. Br Heart f 1993;69:151-7.

18 Pinamonti B, Pagnan L, Bussani R, et al. Right ventricular dysplasia with biventricular involvement. Circulation 1998;98:1943-5.

19 Baraka M, Fontaliran F, Frank R, et al. Value of task force criteria in the diagnosis of arrhythmogenic right ventricular dysplasia/cardiomyopathy. Herzschrittmachertherapie und Elektrophysiologie 1998;9:163-8.

20 Angelini A, Basso C, Turrini P, et al. Clinical and pathological relevance of endomyocardial biopsy. In: Nava A, Rossi L, Thiene G, eds. Arrhythmogenic right ventricular cardiomyopathy/dysplasia. Amsterdam: Elsevier, 1997:25768

21 Blomstrom-Lundqvist C, Sabel KG, Olsson SB. A long term follow up of 15 patients with arrhythmogenic right ventricular dysplasia. Br Heart f 1987; 58:477-88.

22 Pinamonti B, DiLenarda A, Sinagra G, et al. Long term evolution of right ventricular dysplasia/cardiomyopathy. Am Heart f 1995;129:412-15.

23 Wichter T, Borggrefe M, Hawerkamp W, et al. Efficacy of antiarrhythmic drugs in patients with arrhythmogenic right ventricular disease. Circulation 1992;86:29-37.

24 Wichter T, Bocker D, Borggrefe M, et al. Cardioverter-defibrillator therapy. In: Nava A, Rossi L, Thiene G, eds. Arrhythmogenic right ventricular cardiomyopathy/dysplasia. Amsterdam: Elsevier, 1997:376-91.

\section{STAMPS IN CARDIOLOGY}

\section{Transplantation}

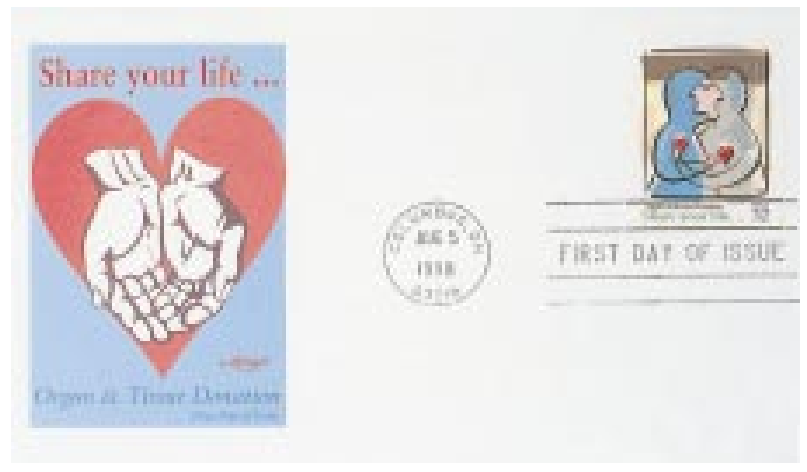

This 32 cent stamp was issued by the United States Postal Service on 5 August at the 1998 National Transplant Games held at Ohio State University in Columbus. Heart transplantation is shown on the stamp which is inscribed "Organ \& Tissue Donation. Share your life". On the reverse of the cover, details are given regarding the organisation of transplantation services including the National Network for Organ Sharing (UNOS). Public information relating to the process for organ and tissue donation is given, and included is the information that "it is possible to donate eight organs from one body: two kidneys, two lungs, one heart, one liver, one pancreas, and intestine".

M K DAVIES A HOLLMAN 